\title{
Stage Classification of Alzheimer's disease in MR Brain Image using Hybrid Clustering Technique and Ensemble Classifier
}

\author{
P.Rajesh kumar, T.Arun Prasath, M.Pallikonda Rajasekaran, G.Vishnuvarathanan
}

\begin{abstract}
Alzheimer's Disease (AD) can be identified in the structural variation of MR brain image. Accurate segmentation of brain subjects is an essential requirement to the quantitative analysis for the pre-surgical planning. Brain network takes place a vital role in the staging of $A D$. Cortical thickness, hippocampus volume and the volume reduction in Grey Matter (GM) have been taken so far in the discrimination of $A D$ subjects from the normal control. The segmentation task has been performed for grouping various brain tissues using Modified Fuzzy C Means algorithm (MFCM) with Particle Swarm Optimization (PSO) and this optimization technique very useful to enhance the segmentation accuracy. Volumetric measure of all the segmented brain tissues have been preferred as one of the feature as well as some structural features also have obtained using Grey Level Co-occurrence Matrix (GLCM) to improve the performance of classifier. In this study, ensemble of classifier has been implemented to classify the severity of the disease. An ensemble of classifier works depends upon the majority voting principle and the error rate of classifier is reduced when compared to the base classifier. The performance of proposed classifier has compared with the base classifiers and verified that the accuracy is improved.
\end{abstract}

Keywords : Alzheimer's disease, Fuzzy C Means, Particle swarm optimization, Grey Level Co-occurrence Matrix.

\section{INTRODUCTION}

Alzheimer's Disease is insistent of neurological brain syndrome which generally initiates slowly and develops worse in the course period of years. The earlier symptoms of $\mathrm{AD}$ are difficulty to progress the memory and complexity like losses in memory, poor judgment, and direction identification, inability to carry out usual works. According to the structural variation only the radiologists could predict the persistence of disease. The purpose of brain MR image segmentation is to allocate each voxels or pixel to explicit tissue class. [5] MR images have some benefits when compared to other modalities such as precise projection of various dimensions, multi-spectral aspect and higher

\section{Revised Manuscript Received on July 22, 2019}

\footnotetext{
P.Rajesh kumar *, School of Electronics and Electrical Technology Kalasalingam Academy of Research and Education,

Krishnankoil, India. Email: rkrrajesh74@ gmail.com

T.Arun Prasath, School of Bio and Chemical Engineering

Kalasalingam Academy of Research and Education

Krishnankoil, India. Email: arun.aklu@gmail.com

M.Pallikonda Rajasekaran, School of Electronics and electrical

TechnologyKalasalingam Academy of Research and Education

Krishnankoil, India. Email: m.p.raja@klu.ac.in

G.Vishnuvarathanan, School of Bio and Chemical Engineering

Kalasalingam Academy of Research and Education

Krishnankoil, India. Email: gvvarthanan@gmail.com
}

resolution for all kind of brain tissues. [6] Variation in the tissues region is a key factor in the analysis of disease severity. Over the past decade some segmentation algorithms have been proposed for accurate segmentation of brain matters. However, due to inhomogeneity of pixel intensity and noise occurrence, the segmentation task is challenging in biomedical imaging applications. FCM algorithm provides additional reports about brain input compared to hard clustering algorithms. In this research work, we have made some modification in the membership function of FCM to get the spatial information from the original input brain image. Cluster center selection process is done with MFCM algorithm and to find out the exact centroid, PSO algorithm also has adopted with this algorithm. PSO provides better results iteratively and enhance the performance of MFCM algorithm. In order to improve the classifier efficiency, various types of features have been selected using GLCM method. Few of the base classifiers are combined and designed an ensemble of classifier to classify the AD which works based on majority voting concept. The results obtained by the ensemble classifier is developed the performance of proposed classifier than base classifiers. [1] Daniel.S., et al have proposed voxel based morphometric for classifying the AD automatically to the input ADNI (Alzheimer's Disease Neuroimaging Initiative) dataset image. Various features have been consisted from the volume of segmented brain structure. The classification accuracy has compared with statistical parameter mapping and proved that is provided better results. [2] Silvia.B., et al have developed a deep learning algorithm for predicting the different stage of $\mathrm{AD}$ affected subjects who have progress from MCI (Mild Cognitive Impairment) to $\mathrm{AD}$. The progress of $\mathrm{AD}$ has been validated with conventional neural network. [3] Jin.L., et al have used edge based features of a particular network to classify the stages of AD. Multiple kernel framework that has used for merging both node based and edge based aspects for staging AD. [4] Gautam.P., et al have evaluated whole brain tractography which is produced from connectivity matrices as well as addition of network measure developed from the flow based measure. The classifier performance has validated with 10 fold cross validation method. [5] G.V.varthanan et al have proposed a hybrid clustering algorithm to differentiate the tissue and tumour region in MR image. Topographical location of exact tumour region has been segmented and the performance of SOM with FKM is validated with standard performance metrics. [6] P.R. Kumar have developed a hybrid segmentation algorithm using $\mathrm{K}$ means with graph cut algorithm to segment brain tissues and essential features have been obtained from that.

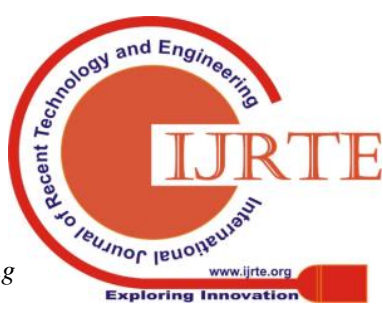




\section{Stage Classification of Alzheimer's disease in MR Brain Image using Hybrid Clustering Technique and Ensemble Classifier}

Game theory based classifier have been implemented to predict the progression of MCI to AD. rectification is not possible.

\section{Methodology}

\section{A. Skull stripping}

Accurate brain extraction is an essential process for achieving successive segmentation of various brain tissues separating non-brain region prior for registration is commonly preferred for facilitates the problem and thus improves the quality of registration. In addition a major problem with brain extraction tool is to achieve sufficient capability while changing the quantity of cerebral atrophy present, as like as due to the presence of AD in one case. Accuracy and robustness of an automated brain extraction is essential for reducing the human intervention. Previous studies comparing few familiar brain extraction methods which show that T1 weighted intensity based skull removal algorithms have some error in segmentation due to a little variation in contrast between CSF region and background. [6] In order to overcome such kind of problems, we have preferred Brain Extraction Tool in our research experiment. BET is the best tool to separate the non-brain region from brain tissue region. It calculates the minimum and maximum brain image pixel intensity value and develops a deformable model to match smoothness of brain surface and regional intensity threshold.

\section{B. Modified FCM}

The FCM algorithm is an advancement of hard K- means clustering principle which is proposed by bezdek. [7] It defines membership class to a data point, based on similarity measure of the data point to a respective class associate to all other classes, this is an iterative optimization task which minimizes objective function. The modified FCM principle is executed for separating various types of brain tissues. The segmentation results are evaluated depending upon the convergence rate and efficiency of segmentation algorithm. The performance of modified FCM algorithm is compared with the traditional FCM and proved that proposed algorithm has provided better results.

$I_{m}(u, v)=\sum_{i=1}^{c} \sum_{j=1}^{n} u_{i j}^{m} d^{2}\left(x_{j}, v_{i}\right)$

With the constrains,

$$
\sum_{i=1}^{c} u_{i j}=1, \quad \forall_{j}
$$

The data matrix $X=\left(x_{1}, x_{2}, \ldots x_{j}, \ldots x_{n}\right)$ with the size of $p \times n, p$ denotes the dimension of each $x_{j}$ and the total number of feature vectors denoted as $n$. [8] The feature vector $X$ is the pixel intensity in the MR image. So, the value of $p=1$ and the member function $u_{i j}$ of the data $j$ in the cluster $i . m$ is a constant which regulates the fuzziness partition regulating. $i^{\text {th }}$ cluster's fuzzy centroid cluster represented as $v_{i *}$ Using Euclidian measure, the distance $d$, computes the similarity between the cluster centroid $v_{i}$ and the feature vector $x_{j}$.

$$
d^{2}\left(x_{j}, v_{i}\right)=\left\|x_{j}-v_{i}\right\|^{2}
$$

When the data points are closer to the centroid vector, then the objective function is minimized and least value of membership function will be neglected. The membership functions are updated using the below formula (4) (5).

$$
\begin{aligned}
u_{i j} & =\left(\sum_{k=1}^{c}\left(\frac{d\left(x_{j}, v_{i}\right)}{d\left(x_{j}, v_{k}\right)}\right)^{2 l(m-1)}\right)^{-1} \\
v_{i} & =\frac{\sum_{j=1}^{n} u_{i j}^{m} x_{j}}{\sum_{j=1}^{n} u_{i j}^{m}}
\end{aligned}
$$

The FCM algorithm iteratively executes two conditions until reaches the objective function. All the data points will be correlated with the membership value, after the FCM clustering for all the classes. The segmentation task could be performed after assigning the highest membership function value to the class of the data point. One of the essential aspect of an image is neighboring pixels have the related feature values belong to the same cluster is considerable. [8] Spatial information is essential in clustering, standard FCM algorithm could not provide it entirely. In order to achieve spatial information, the membership function is modified and given below,

$u_{i j}=\frac{u_{i j}^{m} s_{i j}^{n}}{\sum_{k=1}^{c} u_{k j}^{m} S_{k j j}^{n}}$

Where $S_{i j}=\Sigma_{k \in N\left(x_{j}\right)} u_{i k}$ represents the spatial information, $N\left(x_{j}\right)$ denotes square window placed on pixel $x_{j}$ in spatial domain. $S_{i j}$ denotes the probability that $x_{j}$ belongs to $i^{\text {th }}$ cluster. And $\mathrm{m}$ and $\mathrm{n}$ are parameters. In each iteration, membership function is calculated initially in spatial and according to that spatial function can be calculated.

\section{PSO with MFCM}

The searching process of Particle Swarm Optimization (PSO) that happens based on stochastic search process for the preferred population. The algorithm was developed by Kennedy and Eberhart afterwards monitoring the social interaction [9]. Initially this algorithm is maintaining the population of the particle, the significant solution can be found iteratively for the given optimization problem. The term "swarm" represents total number of possible solution to a particular optimization problem. This technique which determines the particle's perfect location that evaluates the result with specified fitness value. All the particle's positions are defined in the $N_{d}$ search space and that moves among multi-dimensional space. The aim of the algorithm is to obtain nearest position of the particle which has to be very closer to the fitness function. Until find out the best position, the adjustment of position will be updated in each move of the particle. The information of particle's position is maintaining by each particle that has given below,

$x_{i}(t+1)=x_{i}(t)+v_{i}(t+1)$

$x_{i}$-The present position of the particle $v_{i}$-The present velocity of the particle $y_{i}$-The particle's personal best position, by using the above notations, the position adjustment of the particle can be represented as, 


$$
\begin{aligned}
& v_{i, k}(t+1)=v_{i, k}(t)+c_{1} \Gamma_{1, k}(t)\left(y_{i, k}(t)-x_{i, k}(t)\right)+ \\
& c_{2} \Gamma_{2, k}(t)\left(y_{k}(t)-x_{i, k}(t)\right)(8)
\end{aligned}
$$

The inertia weight is $w$, the accelerate on co-efficient are $c_{1}$ and $c_{2}$

Based on three contributions the velocity is calculated,

1. Fraction preceding velocity.

2. The cognitive element of function which is the distance in distinction to the best personal position of the particle.

3. The functional component which is the distance of particle from recently found best position of the particle.

The perfect position of particle is denoted as

$$
y_{i}(t+1)=\left[\begin{array}{lll}
y_{i(t)} & \text { if } & \left.f\left(x_{i}(t+1)\right) \geq f y_{i}(t)\right) \\
x_{i}(t) & \text { if } & \left.f\left(x_{i}(t+1)\right) \leq f y_{i}(t)\right)
\end{array}\right]
$$

The social behavior of particle causes it to get attracted towards best particle in search space. The swarm is separated into overlapping neighbourhoods in $l_{\text {best }}$ PSO model. And the best particle is found for all individual for $g_{b \text { est }}$ PSO model social component equation (7) becomes

$$
c_{2} \Gamma_{2, k}(t)\left(\left(y_{j k}(t)-x_{j k}(t)\right)\right.
$$

Where $y_{j}$ the best particle of the $i^{\text {th }}$ particle in the neighbourhood. Cluster selection task can be executed using FCM algorithm. The mean point of cluster is calculated and that is replaced with the existing cluster, so that the rate of convergence of PSO will improve in each iteration. When the best position identified, that new position will be updated as a cluster center.

\section{Feature selection using GLCM}

In brain MR Image, numerous voxels are presented, even after completed the preprocessing task, still each voxel has not provided any information regarding the disease progression associated with $\mathrm{AD}$. [6] The required features are taken before and after the segmentation task for this research work using Grey Level Co-occurrence Matrix (GLCM) method that calculates angular relationship and distance of subset of the image. Initially GLCM creates the matrix by adding the columns and rows which is equivalent of addition in various subset $N_{g}$. Intensity variation can be estimated using the elements of GLCM for the preferred Region of Interest (ROI). In order to create a GLCM matrix, there are two essential factor are required. The relative angle between two pixels as well as the comparative distance between them. The matrix element $g(i, j)$ which provides the relative frequency of the $i^{\text {th }}$ and $j^{\text {th }}$ elements in the preferred Region of Interest. The angle between the pixels can be measured in various manners such as vertical, horizontal, diagonally at $45^{\circ}$ and $135^{\circ}$.

\section{ENSEMBLE OF CLASSIFIER}

Ensemble of classifier is the combination of few base classifier which categorizes the severity level of disease based on voting principle. For the sake of improving the accuracy of the classifier, combination of classifier has been worked out in this research work and proved ensemble classifier has provided enhanced classification outcomes when compared to base classifier. [10] The base classifier should be distinct in general, it has not exceeded than base classifier. In this classification analysis, some of the base classifiers are based upon majority voting principle and the final decision of ensemble classifier will be based on majority voting from the base classifiers. The base classifier Adaboost, naïve bayes and decision tree are combined as ensemble of classifier.

Ensemble class =

$$
\left\{\begin{array}{cc}
1_{s} & \text { if } \sum_{i=1}^{n} \text { Base classfier class }>\frac{n}{2} \\
-1 & \text { otherwise }
\end{array}\right.
$$

Where, represents no.of classifiers. ' 1 '- denotes AD class ' -1 ' denotes the class of normal subject.

\section{RESULT AND DISCUSSION}

Figure 1 represented the results of proposed MFCM with PSO algorithm has given better outcomes when compared to the previous research works. PSO was very useful to enhance the performance of MFCM algorithm. The images preferred for the research where acquired from the standard dataset Open Access Series Imaging Studies (OASIS) (https://www.oasis-brains.org/). Totally 300 images have been obtained from it in various projections, for training 200 images were used and for testing 100 images were preferred. Normal MR Images also have been involved for the comparative analysis which were obtained from Brain Web database (http://www.bic.mni.mcgill.ca/brainweb/). Fig 1(a) denotes the T1 weighted axial input image with skull region. Generally skull region affects the segmentation results. So, the skull stripping is crucial task in brain related biomedical research applications. To perform skull stripping, various techniques have used in the previous works. Among them Brain Extraction Tool has given preferable outcomes. So, BET was used in this research work. Fig 1(b) mentions the brain MR image without skull region. The input images were analyzed and then proper threshold values have been set in BET tool. The exact brain region has extracted from the input image. Fig 1(c) represents segmentation result of Fast FCM technique. The cluster initialization has done with random manner and uniform sampling manner. So four different cluster has assigned to segment the WM, GM, CSF and hippocampus areas. Fig 1(d) infers the outcomes of K means with PSO technique. In this work also various brain subjects were segmented clearly. Optimization algorithm was used for improving the efficiency of $\mathrm{K}$ means algorithm. Tissues regions were exactly clustered according to intensity variation in the brain image. Fig 1(e) denotes the proposed MFCM with PSO algorithm. The traditional FCM algorithm could not perform well due to the lack of spatial information of the image equation (1) and (2). So, this research have focused on the spatial information of the image, so that the membership function has been modified. The distance between the cluster member and cluster is measured using Euclidian distance metric eqn (3). The membership function update is represented in the eqn (4), (5). Four different clusters were preferred to segment the GM, WM, hippocampus region, CSF. The modification which executed in the membership function is denoted in the eqn (6) for getting the spatial information. In order to improve the clustering results PSO is adopted with the MFCM algorithm. The position update of the particle is represented in the eqn (7), (8). The particles' $l_{\text {best }}$ and $g_{\text {best }}$ positions are updated and mentioned in eqn (9), (10). 
Stage Classification of Alzheimer's disease in MR Brain Image using Hybrid Clustering Technique and Ensemble Classifier

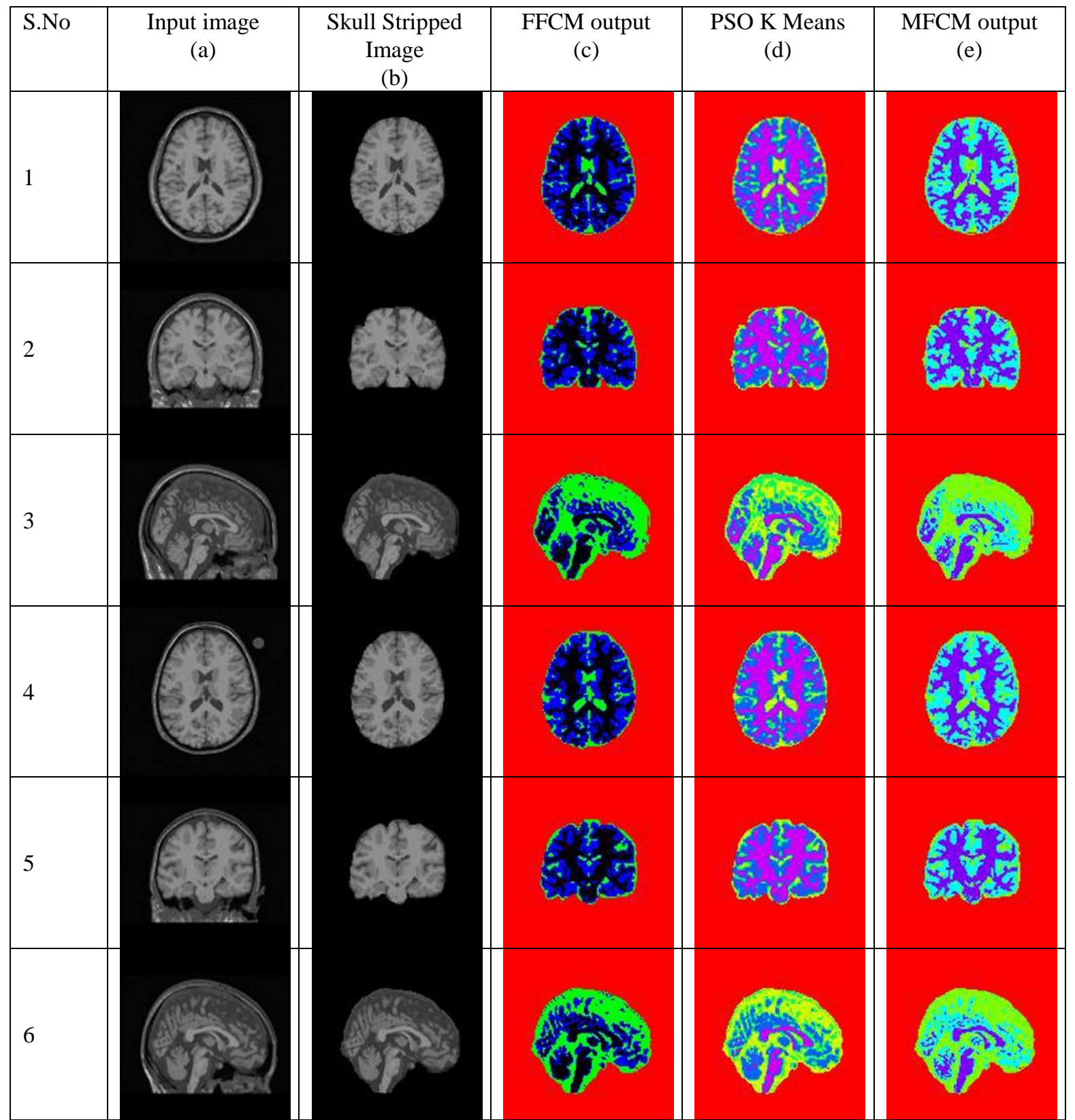

Fig 1(a)-6(a) Input MR images, 1(b)-6(b) MR images without skull, 1(c)-6(c) Results of FFCM algorithm, 1(d)-6(d) Results of PSO K means algorithm, 1(e)-6(e) MFCM with PSO algorithm.

\begin{tabular}{|c|c|c|c|c|c|c|}
\hline Features & Image 1 & Image 2 & Image 3 & Image 4 & Image 5 & Image 6 \\
\hline Entropy & 0.5232 & 0.4827 & 0.5498 & 0.5158 & 0.4904 & 0.5165 \\
\hline Correlation & 0.8734 & 0.8876 & 0.8650 & 0.8776 & 0.8698 & 0.8821 \\
\hline Energy & 0.7653 & 0.7507 & 0.7450 & 0.7821 & 0.7395 & 0.7694 \\
\hline $\begin{array}{c}\text { Cluster } \\
\text { Prominence }\end{array}$ & 2.4323 & 2.2390 & 2.4439 & 2.3367 & 2.3549 & 2.8642 \\
\hline Specificity & 97.6379 & 97.4385 & 97.2534 & 97.7369 & 97.3295 & 97.8548 \\
\hline Sensitivity & 95.2498 & 95.2375 & 95.1945 & 95.2749 & 95.2429 & 95.2565 \\
\hline Recall & 95.6523 & 95.7395 & 95.3641 & 95.7420 & 95.6456 & 95.5863 \\
\hline Accuracy & 96.3427 & 96.5385 & 95.9863 & 96.4869 & 95.9914 & 96.4821 \\
\hline
\end{tabular}

Table 1: Feature selection using GLCM method.

\begin{tabular}{|c|c|c|c|c|c|}
\hline Subjects & Stage & Age & Gender(M/F) & MMSE & CDR \\
\hline 127 & NC & $76.25 \pm 5.42$ & $74 / 53$ & $29.17 \pm 1.08$ & 0 \\
\hline 96 & MCI & $75.42 \pm 7.49$ & $54 / 42$ & $27.02 \pm 1.92$ & 0.5 \\
\hline 73 & AD & $75.63 \pm 8.26$ & $35 / 38$ & $26.18 \pm 2.02$ & 1 \\
\hline \multicolumn{5}{|c|}{ Table 2: Classification of AD/MCI } \\
Retrieval Number: C10331083S219/2019๑BEIESP \\
DOI:10.35940/ijrte.C1033.1083S219
\end{tabular}


Table 1 demonstrates features which were selected using GLCM method. Those are the essential features selected from both post and pre segmentation task. The chosen features are fed as the input to the ensemble of classifier for the classification analysis. The accuracy of the classifier also mentioned which is appreciable when compared to the previous studies. Table 2 represents the progression of MCI to $\mathrm{AD}$. Various age people had monitored for some months and the results were obtained. Among 300 subjects, 127 are normal subjects and 96 are in the stage of MCI and 73 are affected by AD.

\section{CONCLUSION}

This research work mainly focuses on the staging of AD using MFCM with PSO algorithm and ensemble of classifier. Initially segmentation task is executed to separate different types of brain tissues based on the pixel intensity variation in MR brain image using MFCM algorithm. The optimization algorithm supports to enhance the performance of clustering algorithm. Modifications have done in the membership function of the FCM algorithm which could additionally provide the spatial information of the input image. Essential features are selected before and after the segmentation process which support the staging analysis of $\mathrm{AD}$. The disease progression from MCI to AD is classified using ensemble of classification strategy. The classifier results are compared with previous research work and probably proposed classifier performance is improved in the comparative analysis. The neural network based classifier will be adopted in the classifier in future. This research work will be useful to the radiologist in pre-surgical planning of $\mathrm{AD}$.

\section{ACKNOWLEDGMENT}

The authors would like to thank the management of kalasalingam academy of research and education and the school of electronic and electrical technology for providing the computational facilities in VLSI research laboratory.

\section{REFERENCES}

1. Schmitter, D., Roche, A., Maréchal, B., Ribes, D., Abdulkadir, A., Bach-Cuadra, M., Meuli, R. (2015). An evaluation of volume-based morphometry for prediction of mild cognitive impairment and Alzheimer's disease. NeuroImage: Clinical, 7, 7-17.

2. Basaia, S., Agosta, F., Wagner, L., Canu, E., Magnani, G., Santangelo, R., Alzheimer's disease Neuroimaging Initiative. (2019). Automated classification of Alzheimer's disease and mild cognitive impairment using a single MRI and deep neural networks. NeuroImage: Clinical, $21,101645$.

3. Liu, J., Wang, J., Hu, B., Wu, F. X., \& Pan, Y. (2017). Alzheimer's disease classification based on individual hierarchical networks constructed with 3-D texture features. IEEE transactions on nanobioscience, 16(6), 428-437.

4. Prasad, G., Joshi, S. H., Nir, T. M., Toga, A. W., Thompson, P. M., \& Alzheimer's Disease Neuroimaging Initiative (ADNI. (2015). Brain connectivity and novel network measures for Alzheimer's disease classification. Neurobiology of aging, 36, S121-S131.

5. Vishnuvarthanan, G., Rajasekaran, M. P., Subbaraj, P.,Vishnuvarthanan, A. (2016). An unsupervised learning method with a clustering approach for tumor identification and tissue segmentation in magnetic resonance brain images. Applied Soft Computing, 38, 190-212.

6. Kumar, P. R., Arunprasath, T., Rajasekaran, M. P., Vishnuvarthanan, G. (2018). Computer-aided automated discrimination of Alzheimer's disease and its clinical progression in magnetic resonance images using hybrid clustering and game theory-based classification strategies. Computers \& Electrical Engineering, 72, 283-295.
7. Siyal, M. Y., \& Yu, L. (2005). An intelligent modified fuzzy c-means based algorithm for bias estimation and segmentation of brain MRI. Pattern recognition letters, 26(13), 2052-2062.

8. Wang, P., Wang, H. (2008, December). A modified FCM algorithm for MRI brain image segmentation. In 2008 International Seminar on Future BioMedical Information Engineering (pp. 26-29). IEEE.

9. Kumar, P. R., Prasath, T. A., Rajasekaran, M. P., \& Vishnuvarthanan, G. (2017, March). Brain Subject Estimation Using PSO K-Means Clustering-An Automated Aid for the Assessment of Clinical Dementia. In International Conference on Information and Communication Technology for Intelligent Systems (pp. 482-489). Springer, Cham.

10. Farhan, S., Fahiem, M. A., Tauseef, H. (2014). An ensemble-of-classifiers based approach for early diagnosis of Alzheimer's disease: classification using structural features of brain images. Computational and mathematical methods in medicine, 2014. 